\title{
GREEN TENSOR AND REGULAR SOLUTIONS OF EQUATIONS OF RODS THERMODYNAMICS AND THEIR PROPERTIES
}

\author{
Lyudmila A. Alexeyeva, Assyat N. Dadayeva, Nursaule Zh. Ainakeyeva \\ Institute of Mathematics and Mathematical Modelling, Ministry of Education and Science of Kazakhstan, \\ Almaty, Kazakhstan \\ e-mail: alexeeva47@mail.ru; dady1262@mail.ru; nursaule_math@mail.ru
}

\begin{abstract}
In the present paper, solutions of the equations of uncoupled thermoelastodynamics of thermoelastic rods are constructed for power and thermal effects. Based on the Fourier transform, the Green tensor and generalized solutions of the thermoelasticity equations are constructed in the original space-time using the apparatus of generalized functions theory. Analytical formulas for definitions of the thermal stress-strain state of the rods taking into account its thermoelastic parameters are obtained. Shock thermoelastic waves are considered and conditions on their fronts are obtained. The results of numerical calculations of Green tensor are presented.
\end{abstract}

Keywords: thermoelastic rod, Green tensor, thermoelastic wave, stress, temperature

\section{Introduction}

The mechanics of thermoelastic media is currently being intensively developed in the world. The main achievements in this area are related to study of static and quasistatic problems. The most researched solutions are based on the methods of complete and incomplete separation of variables for regions with a canonical form of boundaries. But few studies are devoted to solving dynamic boundary value problems (BVP) of thermoelasticity. The main difficulties are related to the type of dynamic thermoelastic equations. Dynamic problems of thermoelasticity are connected with solving the boundary value problems for mixed hyperbolic-parabolic systems of differential equations, the mathematical theory of which has not yet been sufficiently developed. A complete system of thermoelasticity equations contains hyperbolic equations for medium displacements and a parabolic equation for its temperature (Novatskiy, 1970). They are connected with the gradient of temperature in the first hyperbolic equation and divergence of velocities in the second parabolic one. It leads to presence of singular solutions - shock thermoelastic waves with jumps in stresses and velocities at their fronts (Alipova et al., 2017). To construct solutions of such systems for different external forces and heat sources, the fundamental solution - Green tensor is used. But fundamental solutions of such systems and solutions of BVPs for them in spaces $R^{N}$ $(N=1,2,3)$ were constructed only in spaces of Laplace or Fourier transformation over time (Kupradze et al., 1976; Sharp and Crouch, 1986; Predeleanu, 1987; Ochiai and Ishida, 1988; Sah and Tasaka, 1988; Alexeyeva and Ahmetzhanova, 2018). For construction in their original time-space, numerical methods are used. But they are of little use for studying thermoelastic shock waves.

But by small velocities, it is possible to neglect the divergence of deformation velocity in the heat equation to construct the solutions in the original space. Then we get the equations of theory of thermal stresses (uncoupled thermoelasticity). In papers (Alexeyeva et al., 1999), this model was used for solving the plane BVPs of unsteady thermoelasticity. 
The present paper is devoted to construction of fundamental and regular solutions of uncoupled thermoelasticity in the case $N=1$ in the original space-time by making use of the theory of generalized functions. By slow deformation processes, such solutions describe thermodynamics and the thermally stressed state of thermoelastic rods.

\section{Basic relations of the dynamics of thermoelastic rods}

We consider a thermoelastic rod which is characterized by a linear density $\rho$, the velocity of propagation of elastic waves in the rod, and thermoelastic constants $\kappa, \gamma$. Let us investigate the thermally stressed state of the rod under the influence of external power and heat sources.

The equations of dynamics of the thermoelastic rod have the form (Novatskiy, 1970)

$$
\begin{aligned}
& \rho c^{2} u_{, x x}-\rho u, t t-\gamma \theta,{ }_{x}+\rho F_{1}(x, t)=0 \\
& \theta,{ }_{x x}-k^{-1} \theta_{, t}+F_{2}(x, t)=0
\end{aligned}
$$

Here, $u(x, t)$ are the longitudinal displacements of the cross sections of the rod in the section $x$ at time $t, \theta(x, t)$ the relative temperature $(\theta=T(x, t)-T(x, 0), T$ is the absolute temperature), $F_{1}(x, t), F_{2}(x, t)$ are densities of the power and heat sources of perturbations, respectively

$$
u,_{x}=\frac{\partial u}{\partial x} \quad u,_{x x}=\frac{\partial^{2} u}{\partial x^{2}} \quad u, t=\frac{\partial u}{\partial t} \quad u, t t=\frac{\partial^{2} u}{\partial t^{2}}
$$

The temperature term $\gamma \theta,_{x}$ in the first equation for elastic displacements allows one to take into account the effect of temperature on the stress state of the rod, which is described by the law of Duhamel-Neumann (Novatskiy, 1970)

$$
\sigma(x, t)=\rho c^{2} u,,_{x}-\gamma \theta
$$

Let us construct solutions of equations (2.1) under the action of various power and heat sources of perturbations.

Farther we use the following notation

$$
u_{1}=u \quad u_{2}=\theta \quad u_{i, x}=\frac{\partial u_{i}}{\partial x} \quad u_{i, t}=\frac{\partial u_{i}}{\partial t} \quad i=1,2
$$

Equations (2.1) are of the mixed hyperbolic-parabolic type. Due to hyperbolicity, there are characteristic surfaces $F$ which describe shock thermoelastic waves $F_{t}$.

To get the conditions at fronts of shock waves, we consider Eqs. (2.1) in the space of generalized functions (Vladimirov, 1988). According to the rule of differentiation of a regular discontinuous function, its generalized derivatives are equal to

$$
\begin{aligned}
& \frac{\partial \widehat{u}}{\partial x}=\frac{\partial u}{\partial x}+[u]_{F} \nu_{x} \delta_{F}(x, t) \quad \frac{\partial \widehat{u}}{\partial t}=\frac{\partial u}{\partial t}+[u]_{F} \nu_{t} \delta_{F}(x, t) \quad \nu=\left(\nu_{x}, \nu_{t}\right) \\
& \frac{\partial^{2} \widehat{u}}{\partial x^{2}}=\frac{\partial^{2} u}{\partial x^{2}}+\left[\frac{\partial u}{\partial x}\right]_{F} \nu_{x} \delta_{F}(x, t)+\frac{\partial}{\partial x}\left\{[u]_{F} \nu_{x} \delta_{F}(x, t)\right\} \\
& \frac{\partial^{2} \widehat{u}}{\partial t^{2}}=\frac{\partial^{2} u}{\partial t^{2}}+\left[\frac{\partial u}{\partial t}\right]_{F} \nu_{t} \delta_{F}(x, t)+\frac{\partial}{\partial t}\left\{[u]_{F} \nu_{t} \delta_{F}(x)\right\}
\end{aligned}
$$

By use these formulas, we get from Eqs. (2.1) equations for thermoelastic shock waves in the distribution space

$$
\begin{aligned}
& \rho c^{2} u,{ }_{x x}-\rho u, t t-\gamma \theta,_{x}+F_{1}(x, t)+\left(\nu_{x}\left[\rho c^{2} u,,_{x}-\gamma \theta\right]_{F}-\rho \nu_{t}\left[u,{ }_{t}\right]_{F}\right) \delta_{F}(x, t) \\
& \quad+\rho c^{2} \partial_{x}\left(u[(x, t)]_{F} \delta_{F}\right)-\rho \partial_{t}\left([u(x, t)]_{F} \delta_{F}\right)=0 \\
& \theta,_{x x}-\kappa \theta,{ }_{t}+F_{2}+\partial_{x}\left([\theta(x, t)]_{F} \nu_{x} \delta_{F}\right)+\left(\nu_{x}-\kappa^{-1} \nu_{t}\right)\left[\theta,_{x}\right]_{F} \delta_{F}=0
\end{aligned}
$$


where $\nu=\left(\nu_{x}, \nu_{t}\right)$ is the normal to the characteristic surface $F$ in space $(x, t)$ which satisfies the characteristic equation

$$
\left|\begin{array}{cc}
c^{2} \nu_{x}^{2}-\nu_{t}^{2} & 0 \\
0 & \nu_{x}^{2}
\end{array}\right|=\nu_{x}^{2}\left(c^{2} \nu_{x}^{2}-\nu_{t}^{2}\right)=0
$$

From (2.4) it follows that the shock waves $F_{t}$ move in $R^{1}$ with the speed $c=-\left|\nu_{x}\right| / \nu_{t}$.

To be a solution to Eqs. (2.1), it is necessary that densities of independent layers must be equal to 0 . In virtue of continuity of the medium: $[u]_{F_{t}}=0$. In virtue of independency of the simple layer and derivatives

$$
[\theta]=0 \quad\left[\theta,,_{x}\right]=0 \quad\left[c^{2} u,,_{x} \nu_{x}-u,{ }_{t} \nu_{t}\right]=0
$$

from here, Eqs. (2.5) and (2.2) to follow

$$
[\sigma]_{F_{t}}=-\rho c[\dot{u}]_{F_{t}}
$$

It means that temperature and heat flow are continuous at wave fronts, but stresses have jumps at the wave front, which is proportional to the jump of velocity.

System (2.1) is a closed system for determining the movement and temperature of the rod, given the power and heat sources. Its solution can be evaluated using the fundamental solutions of the thermal conductivity equation and the elastic rod equation. In this case, at first we construct a solution of second equation of system (2.1), then the first equation of elasticity of system (2.1) considering the heat flow in the rod as a mass force, which is determined by the solution of the second equation of system (2.1). This approach is used in the theory of thermal stresses when calculating the thermo-stresses state of frame structures, beams, buildings, bridges and other structures.

Here we will evaluate a different approach based on construction of the Green tensor of connected system (2.1). Knowledge of it allows us not only to build solutions to this system for any forces and heat sources, but also to solve a wide range of non-stationary boundary value problems of rod structures.

\section{Fundamental solutions of the equations of uncoupled thermoelasticity. Green's tensor and its properties}

We shall consider construction of solutions to system (2.1) on the space of generalized vector-functions, whose components belong to the class of generalized functions of slow growth $S^{\prime}\left(R^{2}\right)$ (Alipova et al.. 2017). At first, let us consider the fundamental solutions to system (2.1) under the action of instantaneous concentrated disturbance sources of the form

$$
F_{1}=\delta(x) \delta(t) \delta_{1}^{j} \quad F_{2}=\delta(x) \delta(t) \delta_{2}^{j} \quad j=1,2
$$

here $\delta_{i}^{j}$ is the Kronecker symbol, $\delta(\cdot)$ is the singular delta-function (Vladimirov, 1988).

As the action of the sources $F=\left(F_{1}, F_{2}\right)$ is concentrated at the point $x=0$ at $t=0$, we construct a solution that satisfies the following radiation conditions

$$
\begin{array}{llrl}
U_{i}^{j}(x, t)=0 & t<0 & \forall x \in R^{1} \\
U_{i}^{j}(x, t) \rightarrow 0 & |x| \rightarrow \infty & \forall t>0
\end{array}
$$

Such a fundamental solution is called Green's tensor of thermoelasticity equations. We denote the corresponding matrix of the fundamental solutions by $U_{i}^{j}(x, t)$. 
The components of Green's tensor have the following physical meaning:

- $U_{1}^{1}(x, t)$ is the longitudinal displacement in the rod under the action of an impulsive concentrated force: $F_{1}=\delta(x) \delta(t), F_{2}=0$;

- $U_{1}^{2}(x, t)$ is the longitudinal movement in the rod under the action of a concentrated pulsed temperature source, $F_{1}=0, F_{2}=\delta(x) \delta(t)$;

- $U_{2}^{1}(x, t)$ is temperature of the rod under the action of a pulsed concentrated force: $F_{1}=\delta(x) \delta(t), F_{2}=0$

- $U_{2}^{2}(x, t)$ is temperature of the rod under the action of a concentrated pulsed temperature source: $F_{1}=0, F_{2}=\delta(x) \delta(t)$.

Knowing Green's tensor, we can construct a solution to system (2.1) for any sources in the form of a tensor-functional convolution

$$
u_{j}(x, t)=U_{j}^{k}(x, t) F_{k}(x, t) \quad j, k=1,2
$$

(there is summation by the same indices from 1 to 2 everywhere).

\section{Fourier transforms of matrices of fundamental solutions}

To construct the Green tensor, we use the direct and inverse Fourier transform with respect to $x$ and $t$. For regular generalized functions, it has the form

$$
\begin{aligned}
& \bar{u}(\xi, \omega)=\iint_{R^{2}} u(x, t) \exp [\mathrm{i}(\xi x+\omega t)] d x d t \\
& u(x, t)=\frac{1}{(2 \pi)^{2}} \iint_{R^{2}} \bar{u}(\xi, \omega) \exp [-\mathrm{i}(\xi x+\omega t)] d \xi d \omega
\end{aligned}
$$

Lemma 1. The Fourier transform of the matrices of the fundamental solutions of Eqs. (2.1) has the next form

$$
\begin{aligned}
& \bar{U}_{1}^{1}(\xi, \omega)=\frac{\xi^{2}-\mathrm{i} k^{-1} \omega}{c^{2}\left(\lambda_{1}-\lambda_{2}\right)}\left(\frac{1}{\xi^{2}-\lambda_{1}}-\frac{1}{\xi^{2}-\lambda_{2}}\right) \\
& \bar{U}_{1}^{2}(\xi, \omega)=\frac{\mathrm{i} \widetilde{\gamma} \xi}{c^{2}\left(\lambda_{1}-\lambda_{2}\right)}\left(\frac{1}{\xi^{2}-\lambda_{1}}-\frac{1}{\xi^{2}-\lambda_{2}}\right) \\
& \bar{U}_{2}^{1}(\xi, \omega)=0 \quad \bar{U}_{2}^{2}(\xi, \omega)=\frac{c^{2} \xi^{2}-\omega^{2}}{c^{2}\left(\lambda_{1}-\lambda_{2}\right)}\left(\frac{1}{\xi^{2}-\lambda_{1}}-\frac{1}{\xi^{2}-\lambda_{2}}\right)
\end{aligned}
$$

where $\lambda_{1}=\omega^{2} / c^{2}, \lambda_{2}=\mathrm{i} \omega k^{-1}, \widetilde{\gamma}=\gamma / \rho$.

Proof. Taking into account the properties of the Fourier transform of the derivatives, the Fourier transform $\bar{U}_{i}^{j}(\xi, \omega)$ satisfies the following system of algebraic equations

$$
\begin{aligned}
& -c^{2} \xi^{2} \bar{U}_{1}^{j}+\omega^{2} \bar{U}_{1}^{j}+\mathrm{i} \widetilde{\gamma} \xi \bar{U}_{2}^{j}+\delta_{1}^{j}=0 \\
& -\xi^{2} \bar{U}_{2}^{j}+\mathrm{i} k^{-1} \omega \bar{U}_{2}^{j}+\delta_{2}^{j}=0
\end{aligned}
$$

The solution of system (4.1), according to Cramer's formulas, has the form

$$
\bar{U}_{1}^{j}=\frac{\delta_{1}^{j}\left(\xi^{2}-\mathrm{i} \omega k^{-1}\right)+\mathrm{i} \xi \widetilde{\gamma} \delta_{2}^{j}}{\Delta(\xi, \omega)} \quad \bar{U}_{2}^{j}=\frac{\delta_{2}^{j}\left(\xi^{2} c^{2}-\omega^{2}\right)}{\Delta(\xi, \omega)}
$$


where the determinant $\Delta(\xi, \omega)$ of system (4.3) is equal to

$$
\Delta(\xi, \omega)=c^{2} \xi^{4}-\omega\left(\omega+\mathrm{i} k^{-1} c^{2}\right) \xi^{2}+\mathrm{i} k^{-1} \omega^{3}=c^{2}\left(\xi^{2}-\lambda_{1}\right)\left(\xi^{2}-\lambda_{2}\right)
$$

Its roots have the form

$$
\lambda_{1,2}=\frac{\omega\left(\omega+c^{2} \mathrm{i} k^{-1}\right) \pm \omega \sqrt{\left(\omega+c^{2} \mathrm{i} k^{-1}\right)^{2}-4 c^{2} \mathrm{i} k^{-1} \omega}}{2 c^{2}}
$$

from here we get

$$
\lambda_{1}=\frac{\omega^{2}}{c^{2}} \quad \lambda_{2}=\mathrm{i} \omega k^{-1}
$$

We represent relations (4.4) in a form more convenient for conversion, using decomposition into simpler fractions

$$
\frac{1}{\Delta(\xi, \omega)}=\frac{1}{c^{2}\left(\xi^{2}-\lambda_{1}\right)\left(\xi^{2}-\lambda_{2}\right)}=\frac{1}{c^{2}\left(\lambda_{1}-\lambda_{2}\right)}\left(\frac{1}{\xi^{2}-\lambda_{1}}-\frac{1}{\xi^{2}-\lambda_{2}}\right)
$$

As a result, we obtain the formulas of the lemma. The lemma is proved.

In Fig. 1, the transform and components of the real and imaginary part tensor are shown.

(a)

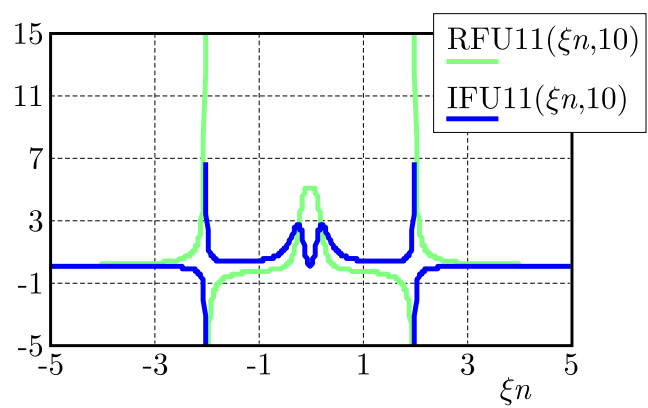

(b)

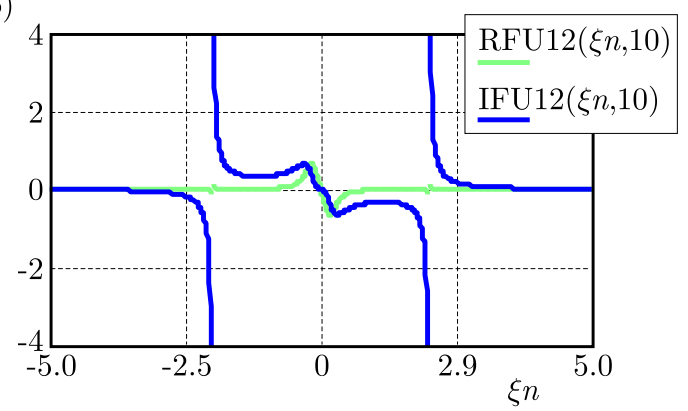

(c)

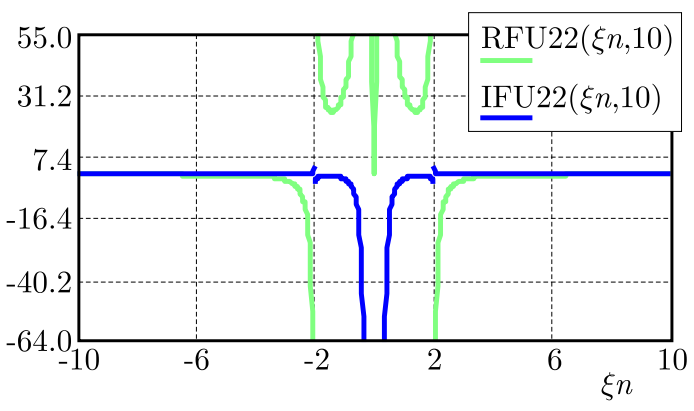

Fig. 1. Real and imaginary parts of $\bar{U}_{i}^{j}(\xi, \omega)(\omega=10,-5<\xi<5)$

\section{Regularization of Fourier transforms of matrices of fundamental solutions. Fourier's transform of Green's tensor}

The Fourier transform (4.4) defines an entire class of fundamental solutions of equations (2.1), which are determined up to the solution of the homogeneous system in the absence of external perturbations. To isolate the Fourier transforms of Green's tensor and their inversion, it is necessary to regularize formulas (4.4) taking into account radiation conditions (3.2). 
Lemma 2. The Fourier transform of Green's tensor of thermoelasticity equations can be represented in the form

$$
\begin{aligned}
\bar{U}_{1}^{1}(\xi, \omega) & =\frac{\xi^{2}-\mathrm{i} k^{-1} \omega}{(\omega+\mathrm{i} 0)\left(\omega-\mathrm{i} c^{2} k^{-1}\right)}\left[\bar{f}_{1}(\xi, \omega)-\bar{f}_{2}(\xi, \omega)\right] \\
\bar{U}_{1}^{2}(\xi, \omega) & =\frac{\mathrm{i} \gamma \xi}{c^{2}\left(\frac{\omega^{2}}{c^{2}}-\mathrm{i} \omega k^{-1}\right)}\left[\bar{f}_{1}(\xi, \omega)-\bar{f}_{2}(\xi, \omega)\right] \\
\bar{U}_{2}^{1}(\xi, \omega) & =0 \quad \bar{U}_{2}^{2}(\xi, \omega)=\frac{(-\mathrm{i} \omega)^{2}-c^{2}(-\mathrm{i} \xi)^{2}}{c^{2}(\omega+\mathrm{i} 0)\left(\omega-\mathrm{i} c^{2} k^{-1}\right)}\left[\bar{f}_{1}(\xi, \omega)-\bar{f}_{2}(\xi, \omega)\right]
\end{aligned}
$$

where

$$
\bar{f}_{1}(\xi, \omega)=\frac{c^{2}}{c^{2} \xi^{2}-(\omega+\mathrm{i} 0)^{2}} \quad \bar{f}_{2}(\xi, \omega)=\frac{1}{\xi^{2}-(\mathrm{i} \omega+\mathrm{i} 0) k^{-1}}
$$

Proof. The difference in the curly brackets of formulas (4.2) contains the Fourier transforms of the fundamental solutions of the d'Alembert equation and the heat equation. Indeed, a function of the form $\bar{f}_{1}(\xi, \omega)=\left[\xi^{2}-\left(\omega^{2} / c^{2}\right)\right]^{-1}$, satisfies the equation: $\left[\xi^{2}-\left(\omega^{2} / c^{2}\right)\right] \bar{f}_{1}=1$, which is the original corresponding to the d'Alembert wave equation for the fundamental solution

$$
\frac{1}{c^{2}} \frac{\partial^{2} f_{1}}{\partial t^{2}}-\frac{\partial^{2} f_{1}}{\partial x^{2}}=\delta(t) \delta(x)
$$

The fundamental solution of this equation, which satisfies the radiation condition, is the well known Riman function (Vladimirov, 1988)

$$
f_{1}(x, t)=\frac{c}{2} H(c t-|x|)
$$

where $H(t)$ is the Heaviside function. Its Fourier transform is a regularization of the right-hand side of a function of the form

$$
\bar{f}_{1}(\xi, \omega)=\operatorname{Reg}\left[\frac{c^{2}}{c^{2} \xi^{2}-\omega^{2}}\right]=\frac{c^{2}}{c^{2} \xi^{2}-(\omega+\mathrm{i} 0)^{2}}
$$

which determines original (5.1) with the carrier on the positive time axis $t$.

Similarly, the second function $\bar{f}_{2}(\xi, \omega)=\left(\xi^{2}-\mathrm{i} \omega k^{-1}\right)^{-1}$ satisfies the equation

$$
\left(\xi^{2}-\mathrm{i} \omega k^{-1}\right) \bar{f}_{2}(\xi, \omega)=1
$$

which in the initial space corresponds to the parabolic heat equation for the fundamental solution

$$
k^{-1} \frac{\partial f_{2}}{\partial t}-\frac{\partial^{2} f_{2}}{\partial x^{2}}=\delta(t) \delta(x)
$$

Its fundamental solution with the carrier on the positive time axis has the form (Vladimirov, 1988)

$$
\begin{aligned}
& f_{2}(x, t)=\frac{H(t) k}{2 \sqrt{\pi k t}} \exp \left(-\frac{x^{2}}{4 k t}\right) \\
& \bar{f}_{2}(\xi, \omega)=\operatorname{Reg}\left[\frac{1}{\xi^{2}-\mathrm{i} \omega k^{-1}}\right]=\frac{k}{k \xi^{2}-\mathrm{i}(\omega+\mathrm{i} 0)}
\end{aligned}
$$

Both functions satisfy the following conditions $(j=1,2)$

$$
\begin{array}{llll}
f_{j}(x, t) & =0 & & t<0 \\
f_{j}(x, t) & \rightarrow 0 & \forall t & |x| \rightarrow \infty
\end{array}
$$

The lemma is proved. 


\section{The Green tensor of thermoelasticity equations}

To construct the original of the Green tensor, we use the properties of the Fourier transform of derivatives and convolutions of generalized functions (Vladimirov, 1988).

Theorem 1. The components of Green's tensor of thermoelasticity equations (2.1) have the form

$$
\begin{aligned}
& U_{1}^{j}(x, t)=\delta_{1}^{j} k^{-1} \frac{\partial \Sigma_{1}}{\partial t}-\delta_{1}^{j} \frac{\partial^{2} \Sigma_{1}}{\partial x^{2}}-\delta_{2}^{j} \widetilde{\gamma} \frac{\partial \Sigma_{1}}{\partial x}-\delta_{1}^{j} \Sigma_{3}(t) \delta(x)+\delta_{2}^{j} \widetilde{\gamma} \frac{\partial \Sigma_{2}}{\partial x} \\
& U_{2}^{j}(x, t)=\delta_{2}^{j} c^{2} \Sigma_{3}(x, t)+\delta_{2}^{j} c^{2} \frac{\partial^{2} \Sigma_{2}}{\partial x^{2}}-\delta_{2}^{j} \frac{\partial^{2} \Sigma_{2}}{\partial t^{2}} \quad j=1,2
\end{aligned}
$$

where

$$
\begin{aligned}
& \Sigma_{1}(x, t)=\frac{k}{c} H(c t-|x|)\left[\frac{k}{c^{2}}\left(1-\mathrm{e}^{\frac{c}{k}(|x|-c t)}\right)+t-\frac{|x|}{c}\right] \\
& \Sigma_{2}(x, t)=-\frac{k^{2} H(t)}{2 c^{2} \sqrt{\pi k}}\left(\mathrm{e}^{-\frac{c^{2}}{k} t} \int_{0}^{t} \frac{1}{\sqrt{\tau}} \mathrm{e}^{\frac{c^{2}}{k} \tau-\frac{x^{2}}{4 k \tau}} d \tau-\int_{0}^{t} \frac{1}{\sqrt{\tau}} \mathrm{e}^{-\frac{x^{2}}{4 k \tau}} d \tau\right) \\
& \Sigma_{3}(x, t)=-\frac{k}{c^{2}} H(t)\left(\mathrm{e}^{-\frac{c^{2}}{k} t}-1\right) \quad \frac{\partial \Sigma_{1}}{\partial t}=-\frac{k}{c} H(c t-|x|)\left(\mathrm{e}^{\frac{c}{k}(|x|-c t)}-1\right) \\
& \frac{\partial \Sigma_{1}}{\partial x}=-\frac{k}{c^{2}} H(c t-|x|) \operatorname{sgn} x\left(\mathrm{e}^{\frac{c}{k}(|x|-c t)}+1\right) \\
& \frac{\partial \Sigma_{2}}{\partial x}=-\frac{A H(t)}{k}\left(\mathrm{e}^{-\frac{c^{2}}{k} t} \int_{0}^{t} \frac{1}{\sqrt{\tau}} \mathrm{e}^{\frac{c^{2}}{k} \tau-\frac{x^{2}}{4 k \tau}} \frac{x}{2 \tau} d \tau-\int_{0}^{t} \frac{1}{\sqrt{\tau}} \mathrm{e}^{-\frac{x^{2}}{4 k \tau}} \frac{x}{2 \tau} d \tau\right) \\
& \frac{\partial^{2} \Sigma_{2}}{\partial t^{2}}=A H(t)\left(\frac{c^{4}}{k^{2}} \mathrm{e}^{-\frac{c^{2}}{k} t} \int_{0}^{t} \frac{1}{\sqrt{\tau}} \mathrm{e}^{\frac{c^{2}}{k} \tau-\frac{x^{2}}{4 k \tau}} d \tau-\frac{c^{2}}{k} \mathrm{e}^{-\frac{c^{2}}{k} t} \frac{1}{\sqrt{\tau}} \mathrm{e}^{\frac{c^{2}}{k} \tau-\frac{x^{2}}{4 k \tau}}\right) \\
& -A H(t)\left[\mathrm{e}^{-\frac{c^{2}}{k} t} \frac{1}{k \sqrt{\tau}} \mathrm{e}^{\frac{c^{2}}{k} t-\frac{x^{2}}{4 k t}}\left(c^{2}-\frac{x^{2}}{4 t^{2}}\right)+\frac{x^{2}}{4 k t^{2}} \frac{1}{\sqrt{\tau}} \mathrm{e}^{-\frac{x^{2}}{4 k t}}\right] \\
& \frac{\partial^{2} \Sigma_{2}}{\partial x^{2}}=\frac{A H(t)}{2 k}\left\{\mathrm{e}^{-\frac{c^{2}}{k} t}\left[\int_{0}^{t} \frac{1}{\tau \sqrt{\tau}} \mathrm{e}^{\mathrm{c}^{2}} \tau-\frac{x^{2}}{4 k \tau}\left(\frac{x^{2}}{2 k \tau}-1\right) d \tau\right]-\int_{0}^{t} \frac{1}{\tau \sqrt{\tau}} \mathrm{e}^{-\frac{x^{2}}{4 k \tau}}\left(\frac{x^{2}}{2 k \tau}-1\right) d \tau\right\}
\end{aligned}
$$

Proof. To restore the original, we note that the following differential operators correspond to the expressions in the numerators of formulas (4.2) in the original space

$$
\begin{aligned}
& \delta_{1}^{j}\left(\xi^{2}-\mathrm{i} \omega k^{-1}\right)+\mathrm{i} \xi \widetilde{\gamma} \delta_{2}^{j} \Longleftrightarrow \delta_{1}^{j}\left(k^{-1} \partial_{t}-\partial_{x} \partial_{x}\right)-\widetilde{\gamma} \delta_{2}^{j} \partial_{x} \\
& \delta_{2}^{j}\left(c^{2} \xi^{2}-\omega^{2}\right) \Longleftrightarrow \delta_{2}^{j}\left(\partial_{t}-c^{2} \partial_{x} \partial_{x}\right)
\end{aligned}
$$

To reconstruct the original denominator in (4.2), we consider

$$
\frac{1}{\omega\left(\omega-\mathrm{i} c^{2} k^{-1}\right)}=\frac{1}{\omega} \times \frac{1}{\left(\omega-\mathrm{i} c^{2} k^{-1}\right)}
$$

For the first factor, we use regularization

$$
\operatorname{Reg}\left[-\frac{1}{\mathrm{i} \omega}\right]=-\frac{1}{\mathrm{i}(\omega+\mathrm{i} 0)} \Leftrightarrow H(t) \delta(x)
$$


The regularization of the second factor, according to the property of the Fourier transform, corresponds to the function

$$
\left[\frac{1}{(\omega+\mathrm{i} 0)-\mathrm{i} c^{2} k^{-1}}\right] \Leftrightarrow g_{1}(t) \delta(x) \quad \text { where } \quad g_{1}(t)=\mathrm{e}^{-\frac{c^{2} t}{k}} H(t)
$$

Now we use the convolution property of generalized functions (Vladimirov, 1988)

$$
h=f * g \quad \Leftrightarrow \quad \bar{h}=\bar{f} * \bar{g}
$$

Consequently, using (5.2) and (5.3) and the convolution property with $\delta$-Dirac's function, we obtain

$$
\begin{aligned}
f_{1}(x, t) * H(t) \delta(x)=f_{1} * H(t) & \Longleftrightarrow \frac{\bar{f}_{1}(\xi, \omega)}{-\mathrm{i}(\omega+\mathrm{i} 0)} \\
f_{2}(x, t) * H(t) \delta(x)=f_{2} * H(t) & \Longleftrightarrow \frac{\bar{f}_{2}(\xi, \omega)}{-\mathrm{i}(\omega+\mathrm{i} 0)}
\end{aligned}
$$

here the symbol $t$ under the convolution sign means convolution only on $t$. Consequently

$$
\begin{aligned}
\operatorname{Reg} & {\left[\frac{1}{\omega} \times \frac{1}{\left(\omega-\mathrm{i} c^{2} k^{-1}\right)}\right]=\frac{1}{-\mathrm{i} \omega} \times \frac{1}{\left(\mathrm{i} \omega+c^{2} k^{-1}\right)} } \\
& \Longleftrightarrow H(t) \delta(x) * g_{1}(t) \delta(x)=H(t) \underset{t}{*} g_{1}(t) \delta(x)
\end{aligned}
$$

Computing these convolutions, we obtain

$$
\begin{aligned}
& H(t) \underset{t}{*} H(t) \mathrm{e}^{-\frac{c^{2}}{k} t}=\int_{-\infty}^{\infty} H(t-\tau) H(\tau) \mathrm{e}^{-\frac{c^{2}}{k} t} d \tau=-\frac{k}{c^{2}} H(t)\left(\mathrm{e}^{-\frac{c^{2}}{k} t}-1\right)=\Sigma_{3}(t) \\
& \Sigma_{3}(t) \delta(x)_{t} * c H(c t-|x|)=-\frac{k}{c} H(t)\left(\mathrm{e}^{-\frac{c^{2}}{k} t}-1\right) * H(c t-|x|)= \\
& =-\frac{k}{c} H(c t-|x|) \int_{|x| / c}^{t}\left(\mathrm{e}^{-\frac{c^{2}}{k}(t-\tau)}-1\right) d \tau \\
& =\frac{k}{c} H(c t-|x|)\left[\frac{k}{c^{2}}\left(1-\mathrm{e}^{\frac{c}{k}(|x|-c t)}\right)+t-\frac{|x|}{c}\right]=\Sigma_{1}(x, t) \\
& \Sigma_{3}(t) \delta(x)_{t} \frac{k H(t)}{2 \sqrt{\pi k t}} \mathrm{e}^{-\frac{x^{2}}{4 k t}}=-\frac{k^{2} H(t)}{2 c^{2} \sqrt{\pi k}}\left(\mathrm{e}^{-\frac{c^{2}}{k} t} \int_{0}^{t} \frac{1}{\sqrt{\tau}} \mathrm{e}^{\frac{c^{2}}{k} \tau-\frac{x^{2}}{4 k \tau}} d \tau-\int_{0}^{t} \frac{1}{\sqrt{\tau}} \mathrm{e}^{-\frac{x^{2}}{4 k \tau}} d \tau\right)=\Sigma_{2}(x, t)
\end{aligned}
$$

In the representation, the Fourier transforms of Green tensor (4.2) in the numerator there are the Fourier transforms of the differential operators

$$
\begin{aligned}
& \delta_{1}^{j}\left(\xi^{2}-\mathrm{i} \omega k^{-1}\right)+\mathrm{i} \xi \widetilde{\gamma} \delta_{2}^{j} \Leftrightarrow \delta_{1}^{j}\left(k^{-1} \partial_{t}-\partial_{x} \partial_{x}\right)-\widetilde{\gamma} \delta_{2}^{j} \partial_{x} \\
& \delta_{2}^{j}\left(c^{2} \xi^{2}-\omega^{2}\right) \Leftrightarrow \delta_{2}^{j}\left(\partial_{t}-c^{2} \partial_{x} \partial_{x}\right)
\end{aligned}
$$

Then

$$
\begin{aligned}
& U_{1}^{j}(x, t)=\left[\left(k^{-1} \frac{\partial}{\partial t}-\frac{\partial^{2}}{\partial x^{2}}\right) \delta_{1}^{j}-\widetilde{\gamma} \delta_{2}^{j} \frac{\partial}{\partial x}\right] \Sigma_{1}(x, t)-\delta_{1}^{j} \Sigma_{3}(t) \delta(x)+\delta_{2}^{j} \widetilde{\gamma} \frac{\partial \Sigma_{2}}{\partial x} \\
& U_{2}^{j}(x, t)=\delta_{2}^{j} c^{2} \Sigma_{3}(x, t)+\delta_{2}^{j} c^{2}\left(\frac{\partial^{2}}{\partial x^{2}}-\delta_{2}^{j} \frac{\partial^{2}}{\partial t^{2}}\right) \Sigma_{2}(x, t)
\end{aligned}
$$


During differentiating the introduced discontinuous functions, taking into account the rules for differentiating such functions in the space of generalized functions (Vladimirov, 1988), we obtain

$$
\begin{aligned}
\frac{\partial \Sigma_{1}}{\partial t} & =\frac{k}{c} \underbrace{\left\{\left[\frac{k}{c^{2}}\left(1-\mathrm{e}^{\frac{c}{k}(|x|-c t)}\right)+t-\frac{|x|}{c}\right]\right\}}_{=0} \delta(c t-|x|)+\frac{k}{c} H(c t-|x|)\left(-\mathrm{e}^{\frac{c}{k}(|x|-c t)}+1\right) \\
& =-\frac{k}{c} H(c t-|x|)\left(\mathrm{e}^{\frac{c}{k}(|x|-c t)}-1\right) \\
\frac{\partial \Sigma_{1}}{\partial x} & =\underbrace{\frac{k}{c}\left[\frac{k}{c^{2}}\left(1-\mathrm{e}^{\frac{c}{k}(|x|-c t)}\right)+t-\frac{|x|}{c}\right]}_{=0}[\delta(x+c t)-\delta(x-c t)] \\
+ & \frac{k}{c} H(c t-|x|)\left(-\frac{k}{c^{2}} \mathrm{e}^{\frac{c}{k}(|x|-c t)} \frac{c}{k} \operatorname{sgn} x-\frac{\operatorname{sgn} x}{c}\right) \\
& =-\frac{k}{c} H(c t-|x|)\left(\frac{1}{c} \mathrm{e}^{\frac{c}{k}(|x|-c t)} \operatorname{sgn} x+\frac{\operatorname{sgn} x}{c}\right)
\end{aligned}
$$

Here the sum of the first two terms is zero; on the support of a simple layer $c t=|x|$ we have

$$
\left[\frac{k}{c^{2}}\left(1-\mathrm{e}^{\frac{c}{k}(|x|-c t)}\right)+t-\frac{|x|}{c}\right]_{|x|=c t}=\frac{k}{c^{2}}\left(1-e^{0}\right)+0=0
$$

Simlarly we get all other derivatives in the formulas of this theorem. As one can see, this tensor is not regular. It contains a singular addend $\delta_{1}^{j} \Sigma_{3}(t) \delta(x)$.

\section{Numerical calculations of the Green tensor. Shock waves}

The program for calculating the Green tensor is elaborated in MathLab system. Calculations of the components of this matrix for different thermoelastic parameters at various points of the medium with conditional thermoelastic parameters are carried out by $\rho=1, c=1, \gamma=1, k=2$.

The program makes it possible to vary the parameters of the problem, which were carried out in numerical experiments to determine their influence on the thermodynamics of the rod. Figures 2a-2f show time variation of the corresponding component of the Green tensor at a fixed point of the rod: $x=1,2,4,10$ and also show a change in the coordinate of the corresponding component of the Green tensor at a fixed time: $t=1,2,4,10$. Here, the jumps of displacements on the graphs of the function are related to the moment of arrival of the elastic shock wave at the point $x_{n}$ at time $t=x n / c$.

To calculate the fundamental stresses, we use Duhamel-Neumann law (2.2).

\section{Determination of thermoelastic displacements, temperature and stresses of the rod under the action of distributed sources}

To determine the displacements in the medium under the action of regular forces and heat sources distributed on $(-L, L)$

$$
F_{1}(x, t)=F(x, t) H(L-|x|) H(t) \quad F_{2}(x, t)=W(x, t) H(L-|x|) H(t)
$$


(a)

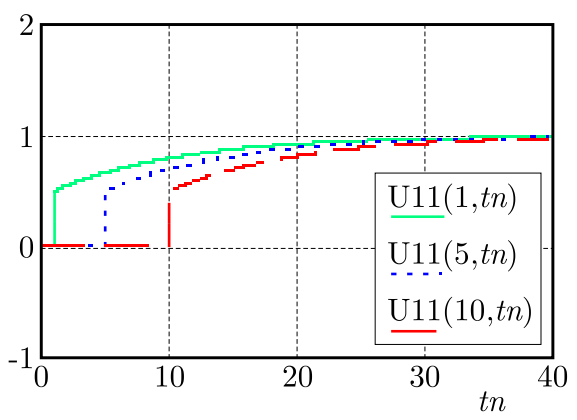

(c)

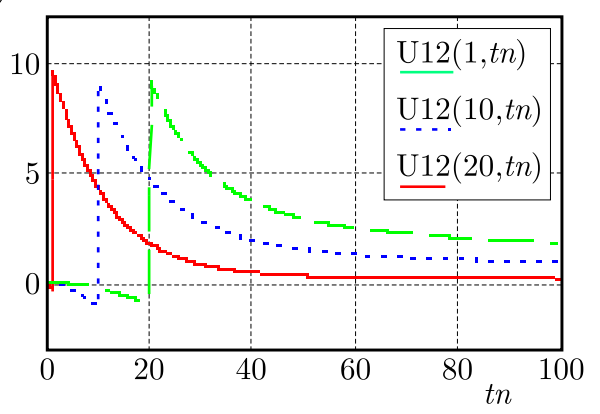

(e)

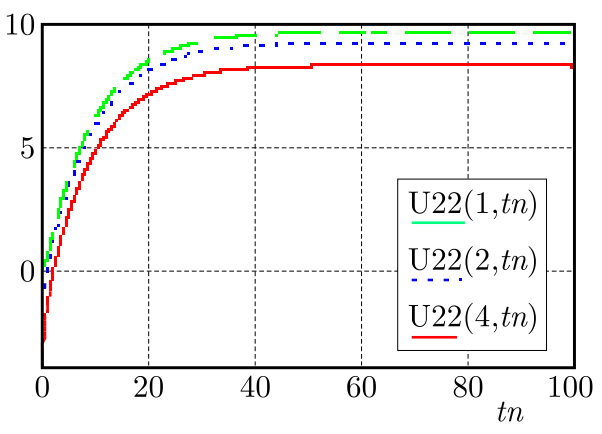

(b)

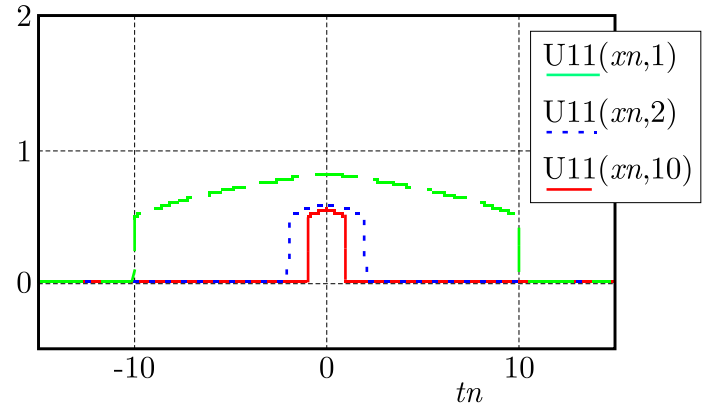

(d)

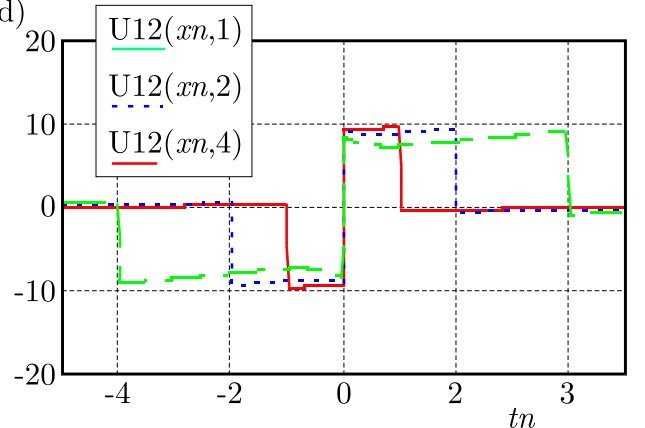

(f)

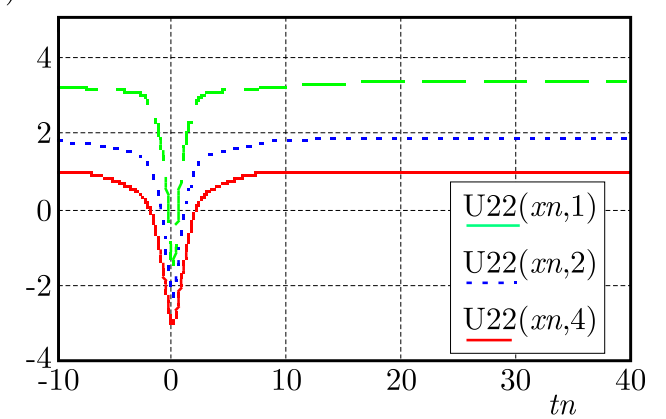

Fig. 2. Green tensor components $U i j(x n, t n)=U_{i}^{j}(x, t)$ at the fixed point and in fixed time

(a)

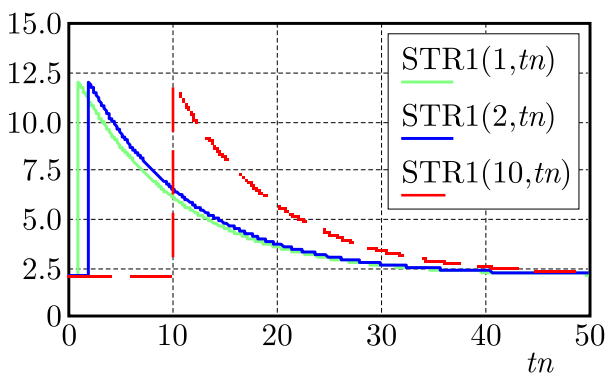

(c)

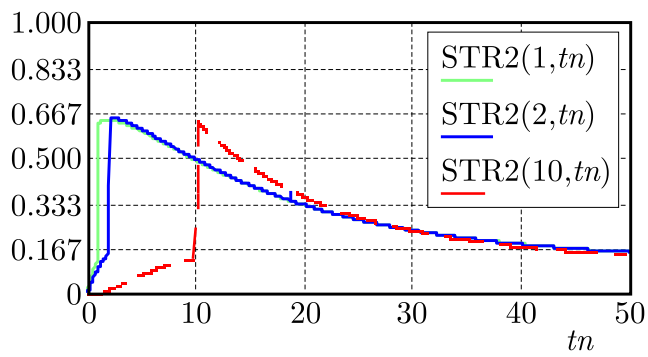

(b)

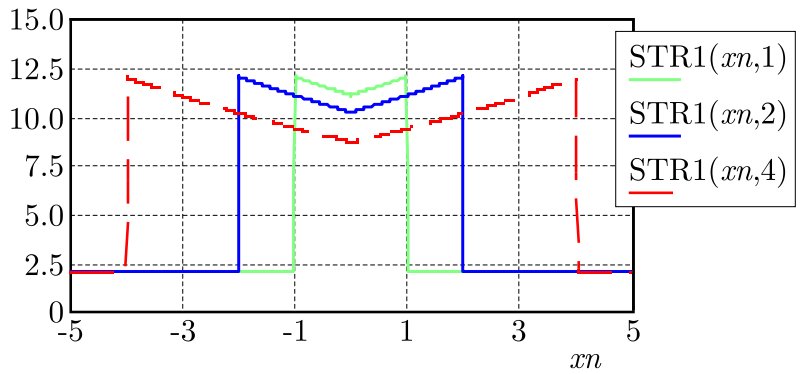

(d)

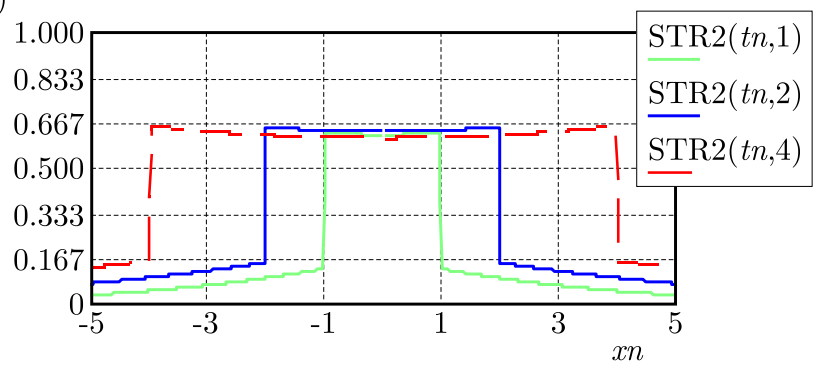

Fig. 3. Thermoelastic stresses under they action of power and heat sources $(a, b, c, d)$ 
we can use the property of convolution with Green's tensor. The displacements and temperature in this case are determined in the integral form

$$
\begin{aligned}
& u(x, t)=H(t) \int_{0}^{t} d \tau \int_{-L}^{L} U_{1 r e g}^{k}(x-y, \tau) F_{k}(y, t-\tau) d y+H(t) \int_{0}^{t} \Sigma_{3}(\tau) F_{1}(x, t-\tau) d \tau \\
& \theta(x, t)=H(t) \int_{0}^{t} d \tau \int_{-L}^{L} U_{2}^{k}(x-y, \tau) F_{k}(y, t-\tau) d y
\end{aligned}
$$

where $U_{1 \text { reg }}^{k}$ is the regular part of the Green tensor. By singular forces and heat sources, the solution of hermoelastodynamics equations (2.1) has the form of the convolutions

$$
u(x, t)=U_{1}^{k}(x, t) * F_{k}(x, t) \quad \theta(x, t)=U_{2}^{k}(x, t) * F_{k}(x, t)
$$

which are calculated according to the definition of convolution in the space of generalized functions.

\section{Conclusion}

In the potential theory, the fundamental solutions are used to construct the solution of BVP by making use of potential of simple and double layers with unknown density, which must be defined from boundary conditions ((Kupradze et al., 1976). The general functions method gives possibility to present solution of BVP over boundary conditions, like Green's formulas. This method for BVPs of thermoelasticity in the Laplace and Fourier transformation space was elaborated in (Alexeyeva and Ahmetzhanova, 2018; Alexeyeva et al., 1999). The constructed here Green's tensor can be used for solving BVPs of uncoupled thermoelasticity in the original space-time by both methods.

The results of this study can be used to assess strength and reliability in the operation of core structures used in engineering and construction of ground and underground facilities (building supports, columns, robotics, etc.)

This work was supported by grants from the Ministry of Education and Science of the Republic of Kazakhstan (No. AP09261033, No. AP05132272).

\section{References}

1. Alekseyeva L.A., Dadayeva A.N., Zhanbyrbaev N.B., 1999, The method of boundary integral equations in boundary value problems of unconnected thermoelastodynamics, Journal of Applied Mathematics and Mechanics, 63, 5, 803-808

2. Alexeyeva L.A., Ahmetzhanova M.M., 2018, Stationary oscillations of thermoelastic rod under action of external disturbances, Global Journal of Engineering Science and Research Management, 5, 2, 33-43, DOI: 10.5281/zenodo.1186513

3. Alipova B.N., Alexeyeva L.A., Dadayeva A.N., 2017, Shock waves as generalized solutions of thermoelastodynamics equations. On the uniquiness of boundary value problems solutions, American Institute of Physics Conference Proceeding, 1798, 020003-1-020003-8, DOI: 10.1063/1.4972595

4. Kupradze V.D., Hegelia T.G., Basheleishvili M.O., Burchuladze T.V., 1976, Three-Dimensional Problems of the Mathematical Theory of Elasticity and Thermoelasticity, Moscow, 664

5. Novatskiy V., 1970, Dynamic Problems of Thermoelasticity, Moscow, Mir, 256 
6. Ochiai Y., Ishida R., 1988, Three-dimensional unsteady thermal stress analyses by means of the thermoelastic displacement potential and boundary element method, Japan Society of Mechanical Engineers Series A, 54, 506, 1847-1850

7. Predeleanu M., 1987, Analysis of thermomechanical coupling by boundary element method, [In:] Thermomechanical Couplings Solids. Jean Mandel Memory Symposium, Amsterdam, 305-318

8. SAH J., TASAKA N., 1988, Boundary element analysis of linear coupled thermoelasticity problems by using Laplace transformation, Proceeding 1st Joint Japan, US Symposium. Boundary Element Methods, Tokyo, 335-544

9. Sharp S., Crouch S.L., 1986, Boundary integral methods for thermoelasticity problems, American Society of Mechanical Engineers (ASME): Journal of Applied Mechanics, 53, 2, 298-302, https://doi.org/10.1115/1.3171755

10. Vladimirov V.S., 1988, Equations of Mathematical Physics, Moscow, Nauka, 511

Manuscript received September 14, 2020; accepted for print January 21, 2021 